\title{
Reducing Teachers' Unfounded Beliefs Through Critical-Thinking Education: A Non-Randomized Controlled Trial
}

\author{
Denis Caroti ${ }^{12}$, Jais Adam-Troian ${ }^{* 3}$, Thomas Arciszewski $^{1}$
}

\begin{abstract}
The prevalence of unfounded beliefs (UB; e.g. supernatural or conspiracy beliefs) remains an important issue due to their negative consequences in various domains. Interventions were shown to reduce supernatural UB only when addressing pseudoscientific beliefs. Based on these findings, we designed a single session intervention aiming to teach participants the epistemological distinction between science and pseudoscience. We then assessed the effectiveness of this intervention. Secondary school teachers $(N=130)$ were assigned to one of two groups focusing on critical thinking with or without the intervention content related to pseudoscience. UB (supernatural, conspiracy, pattern perception) were measured using computerized surveys pre- and one moth post-intervention. Mixed-model analyses revealed the expected decrease in conspiracy $\mathrm{UB}, d=.60$, supernatural $\mathrm{UB}, d=1.01$ and illusory pattern perception, $d=.34$ among teachers in the pseudoscience-focused group. Our intervention constitutes a novel cost-effective tool for critical thinking promotion among education professionals.
\end{abstract}

\footnotetext{
${ }^{1}$ Aix Marseille Univ., Marseille, France

${ }^{2}$ CORTECS team, Marseille, France

${ }^{3}$ Keele University, Newcastle, UK

* Corresponding author : Aix Marseille Univ, LPS, 29 Av. Robert Schuman, 13100 Aix-enProvence, France; troian.jais@ neuf.fr
} 


\section{INTRODUCTION}

Unfounded beliefs (UB, i.e., beliefs that are not supported by available scientific evidence) remain substantially prevalent in our modern societies. For instance, polls conducted across Western countries (US, EU) have found that approximately 10 to 40 percent of the population adheres at least to one form of UB depending on its content (e.g., belief in haunted houses, belief in witches; Gallup, 2005). These beliefs are not limited to the supernatural realm, but can pertain to real-life political events. As an example, several reports highlight a high prevalence of conspiracist beliefs on both shores of the Atlantic Ocean (Gombin, 2013; Chapman, 2017; Ståhl \& van Prooijen, 2018).

The issue with UB is that they can have negative consequences in various domains. It has been known for a long time that irrational health beliefs are predictive of risky health behaviors (Christensen, Moran, \& Wiebe, 1999). In addition, paranormal beliefs are linked with preferences for so-called 'alternative medicine' (Van den Bulck \& Custers, 2009). These, in turn, are responsible for increased mortality rates among cancer patients (Johnson, Park, Gross, \& Yu, 2018). Likewise, exposure to conspiracist beliefs decreases voting, child vaccination, and pro-environmental behavior intentions (Jolley \& Douglas, 2014a) and may encourage individuals to engage in everyday criminal activities through increased perceptions of normlessness (see Jolley, Douglas, Leite, \& Schrader, 2019). Investigating efficient strategies and intervention to combat UB is therefore particularly crucial, especially in the field of education.

Despite the fact that the classroom is a privileged place to implement educational interventions to fight UB among students, the issue of UB (see Van Prooijen, 2017)also extends to teachers. Research suggests that teachers have levels of UB similar to that of the general population (Fuertes-Prieto et al., 2020) and, although there is no direct evidence this might influence students' UB, other types of teachers' beliefs are already known to negatively impact 
students (e.g., teacher's beliefs about students' learning processes, Kane et al., 2010; about bullying, Kochenderfer-Ladd \& Pelletier, 2008). More importantly maybe, teachers' UB might as well affect their decisions to use evidence-based practices in the classroom (e.g. Pashler et al., 2008).

\section{The psychological foundations of unfounded beliefs}

So far, psychological research has identified three broad antecedents to UB: personality, cognition and motivation (Ståhl \& van Prooijen, 2018). Personality traits such as disagreeableness (Swami et al., 2011), schizotypy (Holm, 2009), and paranoid ideation (Fenigstein \& Vanable, 1992; Bruder, Hafke, Neave, Nouripanah \& Imhoff, 2013) are all associated with conspiracist beliefs. Other individual difference variables also considerably influence the individuals' propensity to believe in UB. For instance, tendencies to rationalize societal hierarchy are linked with increased inclinations towards religious belief (Jost, Hawkins, Nosek, Hennes, Stern, Gosling, \& Graham, 2014; see also Whitson, Galinsky, \& Kay, 2015). Similarly, UB of the conspiracy kind correlate with individual differences in social dominance orientation (Sidanius \& Pratto, 2001) as well as with right-wing authoritarian tendencies (Adorno, Frenkel-Brunswik, Levinson, \& Sanford, 1950; Bruder et al., 2013) and political cynicism (Swami et al., 2011).

Unsurprisingly, the strongest predictors of adherence to one UB is the degree to which individuals adhere to other ones (Darwin, Neave, \& Holmes, 2011; Goertzel, 1994; Sutton \& Douglas, 2014). This holds even in the case of artificially created beliefs (in experimental contexts; Swami et al., 2011). Thus, UB are considered to reflect a more general type of cognitive style. Though UB differ in content and do not display perfect correlations among each other, research has extensively investigated and highlighted cognitive factors that constitute a 'fertile ground' for such beliefs. Consequently, it was found that UB stem from similar basic processes such as the tendency to perceive patterns in random noise (see Van Prooijen, Douglas, 
\& De Inocencio, 2017) and are negatively associated with analytical thinking capabilities (Pennycook, Cheyne, Seli, Koehler, \& Fugelsang, 2012; Swami, Voracek, Stieger, Tran, \& Furnham, 2014). Analytical reasoning predicts lower adherence to UB (e.g., Hergovich \& Arendasy, 2005) and is a critical mediator which explains the negative effect of education on UB (Van Prooijen, 2017). Conversely, intuitive reasoning is positively associated with UB (Svedholm \& Lindeman, 2013).

Ample evidence shows that, if stable cognitive and personality-related factors increase the propensity to adhere to UB, these interact with motivational states, which explains why the same individual might display different levels of UB due to contextual changes. Accordingly, three types of motivations underlie adherence to conspiracist beliefs, namely, (a) epistemic, (b) existential (the need to feel safe and in control), and (c) social (the need to belong with a group, see Douglas, Sutton, \& Cichocka, 2017 for an overview). Empirical findings support this classification because adherence to UB is positively associated with uncertainty reduction (Marchlewska, Cichocka, \& Kossowska, 2018), loss of control (Whitson \& Galinsky, 2008), death anxiety (Newheiser, Farias, \& Tausch, 2011), and social exclusion threats (Graeupner \& Coman, 2017).

\section{Fighting unfounded beliefs with psychological interventions}

This knowledge of the psychological determinants of UB has been used to design interventions aiming to reduce individual adherence to them, specifically in educational settings. Since personality-related factors are stable and hard to change through educational interventions, these interventions mostly target three classes of UB predictors : attitudes (UB themselves using persuasion), motivational states (rationality motives) and cognitive capabilities (analytical reasoning, through so-called 'critical thinking' promotion).

One way to target UB directly is to use persuasive 'inoculation' techniques (counter arguments; Compton \& Pfau, 2005; Pfau et al., 1997; see also Papageorgis \& McGuire, 1961). 
To counter UB, providing individuals with counter arguments to UB beforehand will increase the likelihood that they will use those to critically assess UB and defend themselves against future attempts from other individuals to persuade them into adhering to UB. In line with this, several studies have demonstrated the effectiveness of inoculating individuals against UB (for a summary see Dyer \& Hall, 2019). For instance, a video-based intervention among college students in an introductory communication course successfully inoculated them against political UB and fake news (Banas and Miller, 2013). These results also hold among samples from the general public (Braddock, 2019; Roozenbeek, \& van der Linden, 2019) and extend to medical UB (anti-vaccine conspiracy beliefs, Jolley and Douglas, 2014b; Study 2; Jolley \& Douglas, 2017). Practical issues, however, impede the use of such techniques in the classroom. First, the counterarguments must be provided before actual exposure to UB. Second, inoculation relies on permanently updated content - tailored for each kind of UB - to be successful.

Therefore, another approach to fighting UB has relied on targeting motivational states, because they are more general and not constrained by the actual content of each UB. For instance, highly analytical individuals display even higher rates of UB if they are motivated to defend their worldviews (Kahan, Peters, Dawson, \& Slovic, 2017). In line with this, crosssectional investigations showed that cognitive ability negatively predicts adherence to UB mostly among individuals who value their epistemic rationality (Ståhl \& van Prooijen, 2018). Experimental evidence showed a simple rationality prime was capable on increasing the negative association between cognitive ability and UB among secondary school teachers (Troian, Caroti, Arciswzeski, \& Ståhl, 2019). If motivational states facilitate reduction of adherence to UB, we must note a lack of substantial main effects of motivation on UB (with a notable exception in the context of conspiracy beliefs, see Bonetto, Troian.Varet, Lo Monaco, 
\& Girandola, 2018), which makes motivation alone an insufficient basis to build impactful interventions.

Since cognitive factors play a direct role in shaping adherence to all sorts of UB, a successful intervention targeting them should also have a protective effect for a wide range of UB. Several interventions aiming to promote critical thinking skills were successful in reducing college students UB (mostly in the pseudoscientific and supernatural domains; Wesp \& Montgomery 1998; Franz \& Green 2013; Garrett \& Weeks, 2017). These interventions relied mostly on extensive courses addressing skills such as reasoning capabilities or scientific literacy (see Kane, Core, \& Hunt, 2010 for a review). Yet, other similar studies reported no such effect (Dougherty 2004; Kane et al., 2010; McLean and Miller 2010; McLaughlin and McGill 2017). In an attempt to resolve this apparent contradiction, Dyer \& Hall (2019) showed that critical thinking promotion worked using a large sample of participants $(\mathrm{N}>800)$, but only among those having received training that directly addressed pseudoscience and how to detect it.

In sum, interventions relying on critical thinking display robust effects if addressing the issue of pseudoscience upfront. This maybe because cognitive biases and reasoning errors vanish when problems (information) are contextualized (Schustek, Hyafil, \& Moreno-Bote, 2019; see also Gigerenzer, \& Gaissmaier, 2011). Hence, 'contextualizing' critical thinking in the domain of pseudoscience could facilitate pseudoscientific UB change. In addition, other UB like conspiracy UB (which are 'unlikely' but 'possible'; since political conspiracies do occur sometimes), may seem less far-fetched than supernatural UB. For this reason, critical thinking applied to political material might appear to participants as more partisan and trigger corresponding biased processing of the intervention itself (Ditto et al., 2019). Introducing critical thinking by using pseudoscience might avoid these defensive reactions and thus lead to an effect on subsequent conspiracy UB. This may explain the efficacy of psychology methods courses (which often include pseudoscience-related examples) in improving students' statistical 
reasoning (see Nisbett et al., 1987), and UB applied to various domains in general. Amongst all the different strategies discussed above, promoting critical thinking skills - using pseudoscience related content - therefore remains the most generalizable and effective pedagogical strategy to fight UB.

\section{The present study}

We designed a study to assess the efficacy of an intervention aiming to promote critical thinking, and to circumvent some of the problematic aspects highlighted in previous research. To do so, we conducted a conceptual replication (Crandall \& Sherman, 2016) and extension of Dyer \& Hall (2019), who investigated the effects of a one-semester pseudoscience instruction course on supernatural and conspiracy UB among college students. Importantly, our study differed from Dyer \& Hall (2019) on three main aspects.

First, we used a 'control' intervention in which critical thinking skills was also delivered but without reference to pseudoscience. Indeed, Dyer \& Hall (2019) demonstrated the efficacy of pseudoscience instruction (which included both critical thinking skills and pseudosciencerelated content) over a research methods and a comparison group of general education courses (which contained neither).

Second, we designed the protocol so that only one training day should suffice to generate a substantial decrease in UB. To do so, we had to make important changes compared to the intervention from Dyer \& Hall (2019). Because of the time constraint (single session), we were not able to give weekly assignments pertaining to different types of UB to participants at the end of each session. Thus, we opted for a different approach. Research shows that epistemic beliefs (beliefs about the nature of knowledge; Greene \& Yu, 2016) are associated with lower UB because they shape individuals' perception of a belief's accuracy and thus predispose them to engage in deeper (more analytical) processing in the face of claims relying on dubious epistemic justifications (e.g., intuition or authority-based, see Garrett \& Weeks, 2014). 
Therefore, we delivered a training that directly targeted participants' epistemic beliefs by addressing the issue of pseudoscience through the prism of scientific epistemology (i.e., differences between scientific knowledge and belief).

Finally, we conducted the study using a sample of French secondary school teachers. In fact, previous interventions targeted students (mostly in higher education settings) or preservice teachers, impeding the generalizability of their results

\section{Hypotheses.}

In line with the observations of Kane et al. (2010) and the results from Dyer \& Hall (2019) we hypothesized that:

a) In the pseudoscience focused group, participants should display lower UB postintervention compared to pre-intervention (H1a). Also, UB scores in this group should be significantly different from those in the non-pseudoscience focused group postintervention (H1b).

b) In the non-pseudoscience focused intervention group - consisting of a critical thinking class not focusing on pseudoscience - no substantial pre-post differences should be observed (H2).

\section{METHODS}

\section{Ethical statement}

The study was conducted in accordance with the 1964 Helsinki declaration (WMO, 1997) and its later amendments, the ethical principles of the French Code of Ethics for Psychologists (CNCDP, 2012), and the 2016 APA Ethical Principles of Psychologists and Code of Conduct (APA, 2017). Data underlying these findings are openly available at https://osf.io/z5c8a/.

\section{Design}

The study consisted of a simple pre-post-test design with one 'treatment' (pseudoscience focused) and one 'control' (non-pseudoscience focused) intervention. Since allocation to one 
or the other was non-random (participation in mandatory training sessions chosen by participants, see procedure), the study was quasi-experimental. Both interventions were delivered through a presentation format, including talks and debates with participants. They lasted a whole day (two times 3 hours with a lunch break and intermediate breaks every hour and a half approximately). Content details can be found in appendix 1 (see supplementary materials).

In the pseudoscience focused group, the critical thinking promotion training with a focus on pseudoscience and epistemic beliefs included, in the following order: definitions of critical thinking, an introduction to critical thinking and intellectual self-defense, analyzing and categorizing information, epistemological distinctions between belief and knowledge, science and pseudoscience (see Lilienfeld, Ammirati, \& David, 2012), as well as an overview of several reasoning and cognitive biases (correlation-causation, optical illusions, illusory correlations...). These topics were illustrated with relevant examples regarding conspiracy theories and pseudoscientific phenomena. At the end of the session, participants were invited to groupdebate on a topic, using the skills that had been taught to them.

Intervention in the non-pseudoscience focused group was designed to address similar topics (especially optical illusions, cognitive biases and information classification) but they were illustrated in the domain of online activities and media. For instance, topics such as partisan or confirmation bias and so- called 'echo chambers' were discussed. By contrast with the intervention group, participants in this control group were introduced to critical thinking in the context of fake news (including conspiracy beliefs), prejudice, stereotypes and social categorization. No mention or focus on pseudoscience was made. Like in the intervention group, participants were invited to perform activities at the end of the session, this time by applying their newly acquired skills to media image analysis and fake news identification. 
Prior to data collection, we conducted a power analysis with GPower (Faul, Erdfelder, Buchner, \& Lang, 2009) to determine the appropriate sample size. We decided to set the expected effect size to $d=0.56$ or $r=0.27$, which is the average effect size of social psychological research on attitudes (see Richard, Bond, \& Stokes-Zoota, 2003; table 1, p.337). Also, we set desired power at $80 \%$ with an $\alpha=.05$. Using two-tail tests (to limit the risk of type I errors), analyses revealed that a minimum of 56 subjects ( 28 per cell) were needed to detect a within-subjects effect with paired t-tests, while 104 subjects (52 per cell) to detect betweensubject differences with independent t-tests. It is this latter sample size that we aimed to reach to ensure optimal power for all our tests.

Our final sample consisted of 130 teachers $(30.7 \%$ male, Mage $=44.6)$. Contrary to our expectations and due to field constraints, they were unevenly distributed across intervention groups ( $N=87$ vs. $N=43$, yielding a difference ratio of approximately 2 ). Though this distribution ensures maximal power for within-subjects tests, it fell short of 9 participants in the control condition to reach the expected $\mathrm{N}=52$ per condition. To assess whether this would be problematic, we conducted a sensitivity power analysis using the same parameters as before but specifying an unbalanced allocation ratio of 2 . It indicated that, to detect between-subject differences with independent t-tests, a minimum of 116 participants was needed with a sample size of at least $\mathrm{N}=39$ in one of the groups and $\mathrm{N}=77$ in the other. Therefore, our unbalanced conditions would have no consequences for the study's power.

\section{Recruitment procedure}

Our target population was French secondary school teachers from an administrative district in France. Indeed, this study was part of a larger research program aiming to assess prevalence of UB among teachers and pupils, as well as to test the efficacy of critical thinking training programs in reducing UB among these two populations. Incidentally, French teachers need a masters' degree level qualification from the same public institution (INSPE) in order to work, 
allowing to hold education level constant in the sample. Teachers were recruited during their participation to mandatory annual training.

In the context of their professional obligations, French secondary teachers must attend annual training classes on topics they chose and register through a computerized system. Upon registration, we knew when teacher training programs would be delivered and with which content. We contacted trainers responsible for all available critical thinking education interventions (applied to media or pseudoscience) and asked them if they wished to disseminate survey links to their trainees. All of them accepted, leading to a total of four pseudosciencerelated (intervention) and two media-related (control) groups. Interventions were delivered by different teams made up of one, two or three trainers. Thus, slight differences in format (and content) occurred due to constraints linked with trainer availability and experience. Also, allocation to the intervention group was not strictly random, though the procedure was doubleblind (neither participants nor trainers were aware of the research hypotheses). The final allocation scheme can be seen in figure 1 .

Upon registration to the course, teachers were asked through the registration system if they consented to participate in a research program aiming to 'evaluate the efficacy of teacher's training. Upon consent, they were invited to complete an online computerized pre-intervention questionnaire (approximately between one and two weeks prior to intervention) and were asked to complete another computerized post-intervention survey one-month post-intervention. 


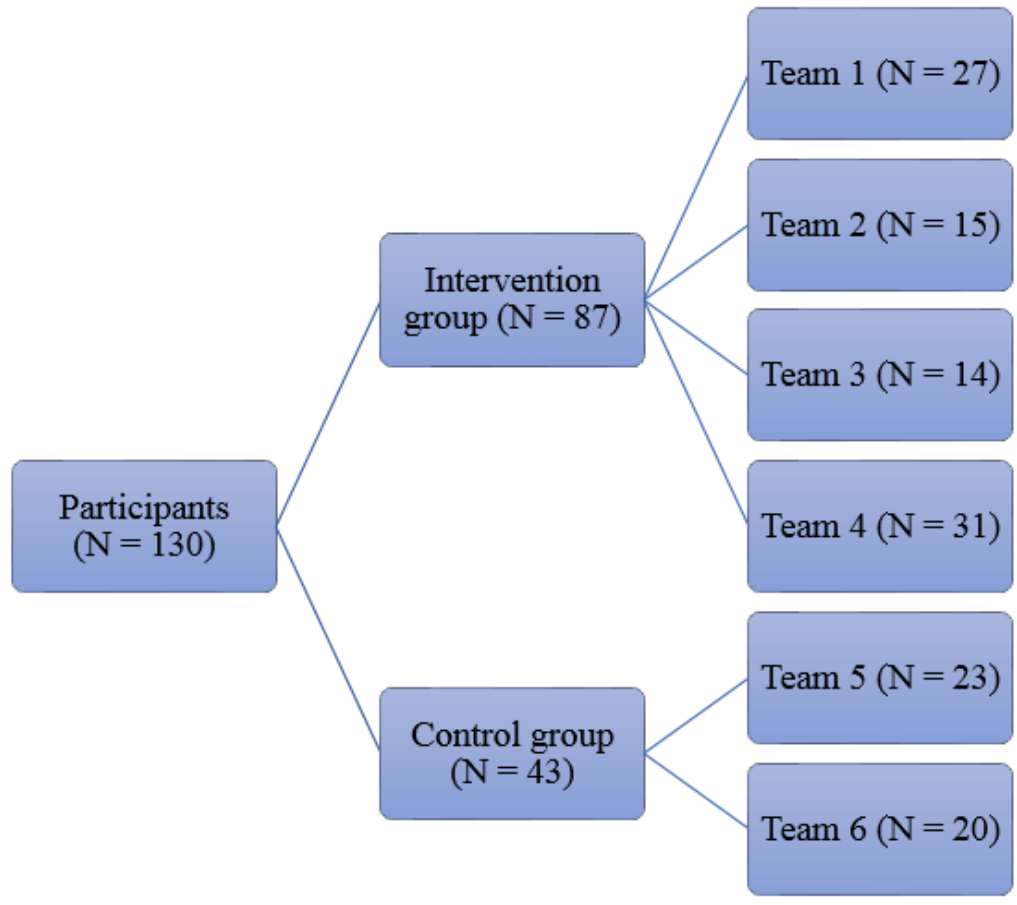

Figure 1. Allocation of participants among intervention groups and training teams.

\section{Materials}

The following measures were introduced to them as measures of personal opinions, personality and cognitive ability:

General Cognitive Ability. General cognitive ability was used as a control variable, to ensure observed differences could not be due to between group pre-intervention differences in cognitive ability. General cognitive ability was thus measured only pre-intervention, using the same Numeracy (Schwartz, Woloshin, Black, \& Welch, 1997) and cognitive reflection tests (CRT, Primi, Morsanyi, Chiesi, Donati, \& Hamilton, 2016) as used by Ståhl \& Van Prooijen (2018, Study 2) and Adam-Troian et al. (2019). In tota,l participants answered 9 cognitive ability items, 3 of which were taken from the numeracy test, and 6 were CRT items. Percentage of correct answers to the 9 items was then computed $(M=61.3, S D=27.8, \alpha=.79)$.

Illusory Pattern Perception task. We decided to include an implicit measure of UB, less sensitive to potential demand characteristic effects. To this end, we drew upon previous research which showed that the tendency to perceive patterns in random stimuli was a common correlate of both conspiracy and supernatural UB (see Van Prooijen, Douglas, and De 
Inocencio, 2017). We used a modified measure of pattern perception adapted from AdamTroian et al. (2019). The original task consisted in rating, for each of 10 series of 10 consecutive dice throws, the extent to which those were completely random or completely determined. An $11^{\text {th }}$ measure was added to those by telling participants that those 10 series were in fact 100 throws with the same dice and asking them to rate the extent to which the results were random or determined. We decided to use only this $11^{\text {th }}$ item to keep survey length low and because it is highly correlated with the rest of the task in previous research (7-point Likert, from 1 'completely random' to 7 'completely determined'; $M=2.45, S D=1.68)$.

Unfounded beliefs. We then asked participants to fill out a French version of the Conspiracist Mentality Questionnaire (Lantian, Muller, Nurra, \& Douglas, 2016). It consists of a series of 5 items for which participants have to assess the likelihood of veracity, and taps into a general conspiracist mindset (see Bruder, Haffke, Neave, Nouripanah, \& Imhoff, 2013; 11point Likert, from $0 \%$ 'completely unlikely' to $100 \%$ 'completely likely'; $M=4.44, S D=1.87$, $\alpha=.81)$.

Finally, we asked participants to fill out a 7-item scale of supernatural beliefs from Adam-troian et al. (2019; adapted from Bouvet, Djeriouat, Goutaudier, Py, and Chabrol, 2014). The scale included items about belief in the existence of various supernatural phenomena such as the existence of the soul, psychokinesis and witchcraft (7-point Likert, ranging from 1 'totally disagree' to 7 'totally agree'; $M=1.97, S D=.84, \alpha=.77$ ).

\section{RESULTS}

\section{Between-group differences pre-intervention}

There were no substantial between group differences in age, $t(128)=.37, p=.72$; gender $\left(\%\right.$ male $e_{\text {intervention }}=29.8 ; \%$ male $\left.e_{\text {control }}=25.6\right), \chi^{2}(1)=.22, p=.64$ and cognitive ability, $t(128)=$ $.38, p=.71$ pre-intervention. Similarly, participants from both groups had comparable preintervention conspiracy UB, $t(128)=.85, p=.40$, supernatural UB, $t(128)=.21, p=.84$ and 
illusory pattern perception, $t(128)=.66, p=.51$ scores. Though participants were not randomly assigned to intervention groups, they had comparable scores on variables critical to the present investigation.

\section{Correlation analyses}

Zero-order correlations between all (standardized) variables can be seen in table 1 . These correlations replicate what is typically found in the literature: cognitive ability was negatively associated with UB measures, and Illusory Pattern Perception. Also, conspiracy UB and Illusory Pattern Perception measures were positively related.

Table 1. Summary of Correlation Analyses Cognitive Ability, Conspiracy Mentality, \begin{tabular}{lllllll} 
Supernatural beliefs and & Illusory Pattern Perception measures across time points $(N=130)$. \\
\hline & 2 & 3 & 4 & 5 & 6 & 7
\end{tabular}

\begin{tabular}{lccccccc}
\hline Cog. Ability & - & - & - & - & - & - & - \\
Conspiracy UB $\mathrm{U}_{0}$ & -.12 & - & - & - & - & - & - \\
Supernatural UB $\mathrm{U}_{0}$ & $-.34 * * *$ & $.32 * * *$ & - & - & - & - & - \\
Illusory Patt.t0 & $-.17 *$ & $.17 *$ & .12 & - & - & - & - \\
Conspiracy UB $\mathrm{U}_{\mathrm{t} 1}$ & -.06 & $.91 * * *$ & $.28 * *$ & $.15^{\dagger}$ & - & - & - \\
Supernatural UB $\mathrm{U}_{\mathrm{t} 1}$ & $-.24 * *$ & $.35^{* * *}$ & $.91 * * *$ & .07 & $.39 * * *$ & - & - \\
Illusory Patt.t1 & -.14 & $.18^{*}$ & .07 & $.68 * * *$ & $.26 * *$ & .11 & - \\
\hline
\end{tabular}

Note. $t 0=$ pre-measures, $t 1=$ post-measures, Numbers represent Pearson correlation coefficients. ${ }^{\dagger} p<.10,{ }^{*} p<.05, * * * p<.001$.

Hypotheses test

Intervention effect. To estimate treatment effect, a linear mixed-model containing one nested random effect (subjects within training groups; see Schielzeth \& Nakagawa, 2013) was computed for each outcome, adjusting for their respective baseline scores to avoid regression to the mean and potential confounding (including them as covariates, see Twisk, Bosman, Hoekstra, Rijnhart, Welten, \& Heymans, 2018) according to the following equations: 
(1) Conspiracy $U B \sim 1+(1 \mid$ subjects $)+(1 \mid$ training group $)+$ time + intervention + intervention:time + baseline-Conspiracy $U B$

(2) Supernatural $U B \sim 1+(1 \mid$ subjects $)+(1 \mid$ training group $)+$ time + intervention + intervention:time + baseline-Supernatural UB

(3) Illusory Pattern Perception $\sim 1+(1 \mid$ subjects $)+(1 \mid$ training group $)+$ time + intervention + intervention:time + baseline-Illusory Pattern Perception

As can be seen in table 2, models for all outcomes displayed satisfying fit indices, and each of them revealed an interaction effect between time and intervention groups, $t(249)=3.21$, $p=.002, d=.60$ for Conspiracy UB, $t(249)=5.46, p<.001, d=1.01$ for Supernatural UB and $t(249)=1.81, p=.072, d=.34$ for Illusory Pattern Perception, albeit marginal this time. Sensitivity analyses revealed that the effects remained stable despite including each outcome's baseline levels as supplementary covariates or excluding all covariates from the models (see appendix 2 in supplementary materials).

Post-hoc tests. Because of the number of tests, we carried out $(\mathrm{N}=9)$, we report adjusted p-values using Holmes correction (see also Aickin \& Gensler, 1996). As can be seen in figure 2, participants in the pseudoscience focused group displayed lower Conspiracy UB, $t(128)=$ $5.70, p<.001, d=.26$, Supernatural UB, $t(128)=7.42, p<.001, d=.34$ and Illusory Pattern Perception, $t(128)=3.85, p=.001, d=.33$ post-intervention compared to pre-intervention. Thus, H1a could not be rejected.

Though displaying comparable scores on all outcomes prior to training, participants in the pseudoscience focused group ended up with lower levels of Conspiracy UB, $t(9)=3.12, p$ $=.054, d=.58$, Supernatural UB, $t(12)=6.75, p<.001, d=1.26$ and Illusory Pattern Perception $t(13)=2.63, p=.084, d=.49$ post-intervention compared to those in the non-pseudoscience focused group. These results provided corroboration for H1b. 
Finally, participants in the non-pseudoscience focused group did not display substantially different levels of Conspiracy UB, $t(128)=.09, p=.99, d=.01$, Supernatural UB $t(128)=1.46, p=.45, d=.09$ and Illusory Pattern Perception $t(128)=.50, p=.99, d=.06$ post-intervention, in line with $\mathrm{H} 2$.

Table 2. Mixed models for intervention effect estimates (Outcomes: Model 1 = Conspiracy UB; Model 2 = Supernatural UB; Model 3 = Illusory Pattern Perception).

\begin{tabular}{|c|c|c|c|c|c|c|c|}
\hline & $t(D f)$ & $A I C$ & $I E E(\mathrm{SE})$ & $95 \% C I$ & $r_{\text {conditional }}^{2}$ & $r_{\text {marginal }}^{2}$ & $p$ \\
\hline Model 1 & & 404.30 & & & .93 & .92 & \\
\hline Time & $3.35(255)$ & & $-.22(.07)$ & {$[-.35,-.09]$} & & & $<.001$ \\
\hline Int. & $1.90(255)$ & & $-.24(.13)$ & {$[-.48, .01]$} & & & .12 \\
\hline Baseline & $54.87(255)$ & & $1.78(.03)$ & {$[.05, .28]$} & & & $<.001$ \\
\hline Int:Time & $3.21(255)$ & & $-.43(.13)$ & {$[-.69,-.17]$} & & & .002 \\
\hline Model 2 & & 7.03 & & & .93 & .92 & \\
\hline Time & $3.08(255)$ & & $-.09(.03)$ & {$[-.15,-.03]$} & & & .002 \\
\hline Int. & $4.27(255)$ & & $-.16(.04)$ & {$[-.23,-.09]$} & & & .012 \\
\hline Baseline & $55.36(255)$ & & $.73(.01)$ & {$[.71, .76]$} & & & $<.001$ \\
\hline Int:Time & $5.46(255)$ & & $-.33(.06)$ & {$[-.45,-.21]$} & & & $<.001$ \\
\hline Model 3 & & 675.11 & & & .74 & .73 & \\
\hline Time & $2.62(255)$ & & $-.30(.11)$ & {$[-.52,-.08]$} & & & .009 \\
\hline Int. & $1.92(255)$ & & $-.26(.13)$ & {$[-.52, .01]$} & & & .12 \\
\hline Baseline & $26.11(255)$ & & $.77(.03)$ & {$[.71, .82]$} & & & $<.001$ \\
\hline Int:Time & $1.81(255)$ & & $-.41(.23)$ & {$[-.86, .03]$} & & & .072 \\
\hline
\end{tabular}

Note . Int. = Intervention Group, Baseline = Outcome Baseline Score, IEE = standardized betas for Intervention Effect Estimate, $\mathrm{SE}=$ Standard Error. ${ }^{\dagger} p<.10,{ }^{*} p<.05,{ }^{* *} p<.01$, $* * * p<.001$. 


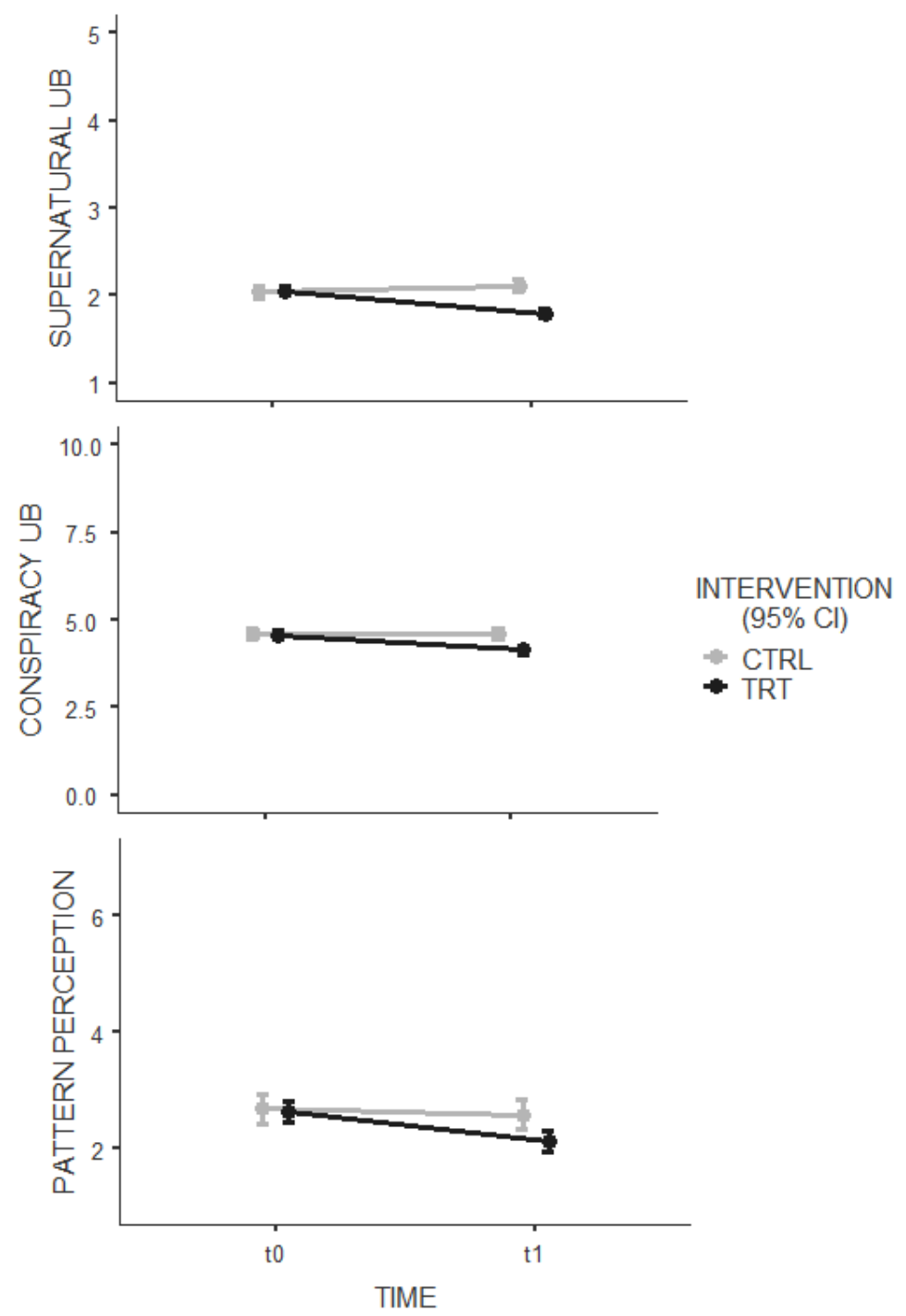

Figure 2. Participants' outcome scores across time and conditions. $\mathrm{t} 0=$ pre-intervention, $\mathrm{t} 1$ = post-intervention, red $=$ non-pseudoscience focused group, blue $=$ pseudoscience focused group. Error bars represent 95\%CIs.

\section{DISCUSSION}

By designing this study, we attempted to replicate the results from Dyer \& Hall (2019), as well as to extend them to both conspiracy UB and Illusory Pattern Perception. Across outcome measures, we demonstrated that a single-day training could substantially lower UB up to a month after intervention. Results were robust to several model specifications and to the inclusion of different related covariates, including analytical thinking scores. Moreover, we successfully designed and implemented an intervention that used previously recommended 
content on pseudoscience, while delivering it with limited real-life examples but with a focus on epistemic beliefs.

Therefore, the viability of our intervention bears some theoretical implications. To the extent that epistemic beliefs are a form of meta-cognition, and that meta-cognitive processes are domain general, they could be a more potent target for intervention. However, given that we obtained the same results than Dyer \& Hall (2019) but using a different pedagogical approach to pseudoscience, one may wonder whether target or content only may matter. Because of field constraints, we were not able to do so, but further investigations should be conducted using both types of pseudoscience related interventions and assessing their relative efficacy compared to equivalent interventions not including pseudoscience as a content.

In addition, for the first time, we used a 'control' group constituted of another type of intervention (instead of giving participants a course related to methods or reasoning in general). In this group, participants were made aware of their biases, and used techniques applied to media-related content to learn how to spot fake news and use critical thinking skills. But this did not lead to any substantial change in their levels of conspiracy beliefs. This is in line with the idea that defensive reactions may occur when introducing critical thinking in the domain of political UB and points at a potent role of contextualization in the realm of pseudoscience. Once the critical thinking skills are successfully acquired by participants, these could be more likely applied in a political context, with less threat perceptions, although further investigation is needed to corroborate this hypothesis (see Jonas et al., 2014).

\section{Limitations}

Nonetheless, the abovementioned points are subjected to important boundary conditions that will now be discussed. First, our sample was specific (French secondary school teachers; mostly female). It is true that robust effects tend to replicate despite considerable heterogeneity across contexts and samples (see Klein et al., 2018), and that these results partly replicate outcomes of 
studies conducted among university students with a different cultural background (AngloSaxon). Also, lowering UB among highly educated individuals should be seen as a strength of the study, since we should expect floor effects among such samples (who already have less UB than the general population; e.g. van Prooijen, 2017) However, caution invites to limit generalizability of these findings to educated Western populations in absence of cross-cultural replications in other contexts (see Henrich, Heine, \& Norenzayan, 2010).

Relatedly, effect sizes for some models were consequential and could be interpreted as reflecting demand characteristics (Orne, 1962). Yet, we believe this was not case because in the group that discussed the issue of fake news and conspiracy theories (non-pseudoscience focused), no reduction in participants' conspiracy UB was observed. Also, the effect on an illusory pattern perception in the intervention group occurred despite this 'bias' not being a discussed in the training.

Yet, it is true that intervention's effect on illusory pattern perception was somehow weaker than for the two other UB outcomes, achieving marginal statistical significance $(p=$ .072 for Model 3's interaction effect). We believe this high p-value results from the observed effect being smaller than the one we expected $(d=.34$ whereas our study was powered for $d$ $=.42$ ), the measure being a single item and the nature of pattern perception itself (lower-level cognitive factors are more resistant to change, Lai et al., 2014).

More importantly, allocation to intervention groups was not random. Even if results were robust across training groups and though intervention groups did not differ on several 'critical' characteristics (such as demographics, analytical thinking and UB prior to intervention), we cannot exclude that other unmeasured factors might constitute confounds. Such factors could be various, ranging from political ideology and extremism (which is related irrational beliefs, Harrington, 2013), to traits such as schizotypy (which is an antecedent of conspiracy UB, Darwin et al., 2011) and future randomized-controlled trials should be 
conducted to replicate our findings. Finally, interventions in the two groups were related, but still differed in various respects, which limits the extent to which we can deduce that exposure to pseudoscientific-related topics only could cause the observed differences.

\section{Conclusion}

Thus, we believe this study constitutes a successful replication and extension of Dyer \& Hall's (2019) results and show that targeted interventions of a few hours can durably affect UB, even in their more implicit form (i.e. Illusory Pattern Perception). Moreover, demand characteristics and confounding are unlikely to provide alternative explanations for the observed pattern. From an applied perspective, we demonstrate that targeting epistemic beliefs (Greene \& Yu, 2016) in critical-thinking promotion could be a cost-effective (time saving) strategy to provide transferrable knowledge that participants use to revise their beliefs and attitudes. Yet, this study lacked a comparison intervention discussing pseudoscience through the prism of bias and ecological examples. This highlights the pressing need for so-called 'dismantling' studies, which are designed to assess the components of interventions responsible for their efficacy (Guidi et al., 2018), in the context of critical-thinking promotion.

\section{REFERENCES}

Adam, A., \& Manson, T. M. (2014). Using a pseudoscience activity to teach critical thinking. Teaching of Psychology, 41(2), 130-134.

Adam-Troian, J., Caroti, D., Arciszewski, T., \& Ståhl, T. Unfounded beliefs among teachers: The interactive role of rationality priming and cognitive ability. Applied Cognitive Psychology. Advanced Online Publication.

Adorno, T. W., Frenkel-Brunswik, E., Levinson, D. J., \& Sanford, R. N. (1950). The authoritarian personality. New York, USA: Harper \& Brothers

Aickin, M., \& Gensler, H. (1996). Adjusting for multiple testing when reporting research results: the Bonferroni vs Holm methods. American journal of public health, 86(5), 726728.

American Psychological Association. (2017). Ethical principles of psychologists and code of conduct. Retrieved from http://www.apa.org/ethics/code/index.aspx

Banas, J. A., \& Miller, G. (2013). Inducing resistance to conspiracy theory propaganda:

Testing inoculation and metainoculation strategies. Human Communication Research, 39(2), 184-207.

Bonetto, E., Troian, J., Varet, F., Lo Monaco, G., \& Girandola, F. (2018). Priming resistance 
to persuasion decreases adherence to conspiracy theories. Social Influence, 13(3), 125136.

Bouvet, R., Djeriouat, H., Goutaudier, N., Py, J., \& Chabrol, H. (2014). Validation française de la Revised Paranormal Belief Scale. L'Encéphale, 40(4), 308-314.

Braddock, K. (2019). Vaccinating Against Hate: Using Attitudinal Inoculation to Confer Resistance to Persuasion by Extremist Propaganda. Terrorism and Political Violence, Advanced Online Publication.

Bruder, M., Haffke, P., Neave, N., Nouripanah, N., \& Imhoff, R. (2013). Measuring individual differences in generic beliefs in conspiracy theories across cultures: Conspiracy mentality questionnaire. Frontiers in Psychology, 4, 225.

Chapman University (2017). Paranormal America. Retrieved from https://blogs.chapman.edu/wilkinson/2017/10/11/paranormal-america-2017/

Christensen, A., Moran, P., \& Wiebe, J. (1999). Assessment of Irrational Health Beliefs. Health Psychology, 18(2), 169-176.

Commission Nationale Consultative de Déontologie des Psychologues (2012). Code de deontologie des psychologues (1996, Amended February, 2012). Retrieved from http://www.cncdp.fr/index.php/code-de-deontologie/code-de-deontologie-2012

Compton, J. A., \& Pfau, M. (2005). Inoculation theory of resistance to influence at maturity:

Recent progress in theory development and application and suggestions for future research. Annals of the International Communication Association, 29(1), 97-146.

Crandall, C. S., \& Sherman, J. W. (2016). On the scientific superiority of conceptual replications for scientific progress. Journal of Experimental Social Psychology, 66, 9399.

Darwin, H., Neave, N., \& Holmes, J. (2011). Belief in conspiracy theories: The role of paranormal belief, paranoid ideation and schizotypy. Personality and Individual Differences, 50, 1289-1293.

Ditto, P. H., Liu, B. S., Clark, C. J., Wojcik, S. P., Chen, E. E., Grady, R. H., ... \& Zinger, J. F. (2019). At least bias is bipartisan: A meta-analytic comparison of partisan bias in liberals and conservatives. Perspectives on Psychological Science, 14(2), 273-291.

Dougherty, M. J. (2004). Educating believers: Research demonstrates that courses in scepticism can effectively decrease belief in the paranormal. Skeptic, 10(4), 31-35.

Douglas, K. M., Sutton, R. M., \& Cichocka, A. (2017). The psychology of conspiracy theories. Current directions in psychological science, 26(6), 538-542.

Dyer, K. D., \& Hall, R. E. (2019). Effect of critical thinking education on epistemically unwarranted beliefs in college students. Research in Higher Education, 60(3), 293-314.

Faul, F., Erdfelder, E., Buchner, A., \& Lang, A. G. (2009). Statistical power analyses using $\mathrm{G}^{*}$ Power 3.1: Tests for correlation and regression analyses. Behavior research methods, 41(4), 1149-1160.

Fenigstein, A., \& Vanable, P. A. (1992). Paranoia and self-consciousness. Journal of personality and social psychology, 62(1), 129. 
Franz, T. M., \& Green, K. H. (2013). The Impact of an Interdisciplinary Learning Community Course on Pseudoscientific Reasoning in First-Year Science Students. Journal of the Scholarship of Teaching and Learning, 13(5), 90-105.

Fuertes-Prieto, M. Á., Andrés-Sánchez, S., Corrochano-Fernández, D., Urones-Jambrina, C., Delgado-Martín, M. L., Herrero-Teijón, P., \& Ruiz, C. (2020). Pre-service Teachers' False Beliefs in Superstitions and Pseudosciences in Relation to Science and Technology. Science \& Education, 29(5), 1235-1254.

Gallup US (2005). Paranormal Beliefs Come (Super)Naturally to Some. Retrieved from https://news.gallup.com/poll/19558/paranormal-beliefs-come-supernaturallysome.aspx

Garrett, R. K., \& Weeks, B. E. (2017). Epistemic beliefs' role in promoting misperceptions and conspiracist ideation. PLOS ONE, 12(9), e0184733.

Gigerenzer, G., \& Gaissmaier, W. (2011). Heuristic decision making. Annual review of psychology, 62, 451-482.

Goertzel, T. (1994). Belief in conspiracy theories. Political Psychology, 731-742.

Goldstein, D. G., \& Gigerenzer, G. (2002). Models of ecological rationality: the recognition heuristic. Psychological review, 109(1), 75.

Gombin, J. (2013). Conspiracy theories in France. Interim report. Retrieved from

Counterpoint website:

http://web.archive.org/web/20140308021114/http://counterpoint.uk.com/wpcontent/uploads/2 013/05/Conspiracy-Theories-in-France-interim-report-3rd-May.pdf

Graeupner, D., \& Coman, A. (2017). The dark side of meaning-making: How social exclusion leads to superstitious thinking. Journal of Experimental Social Psychology, 69, 218222.

Greene, J. A., \& Yu, S. B. (2016). Educating critical thinkers: The role of epistemic cognition. Policy Insights from the Behavioral and Brain Sciences, 3(1), 45-53.

Guidi, J., Brakemeier, E. L., Bockting, C. L., Cosci, F., Cuijpers, P., Jarrett, R. B., ... \& Rief, W. (2018). Methodological recommendations for trials of psychological interventions. Psychotherapy and psychosomatics, 87(5), 276-284.

Harrington, N. (2013). Irrational beliefs and socio-political extremism. Journal of RationalEmotive \& Cognitive-Behavior Therapy, 31(3), 167-178.

Henrich, J., Heine, S. J., \& Norenzayan, A. (2010). The weirdest people in the world? Behavioral and brain sciences, 33(2-3), 61-83.

Hergovich, A., \& Arendasy, M. (2005). Critical thinking ability and belief in the paranormal. Personality and Individual Differences, 38, 1805-1812.

Holm, N. (2009). Conspiracy theorizing surveillance: considering modalities of paranoia and conspiracy in surveillance studies. Surveillance \& Society, 7(1), 36-48.

Johnson, S. B., Park, H. S., Gross, C. P., \& Yu, J. B. (2017). Use of alternative medicine for cancer and its impact on survival. JNCI: Journal of the National Cancer Institute, 110(1), 121-124.

Jolley, D., \& Douglas, K. M. (2014a). The social consequences of conspiracism: Exposure to conspiracy theories decreases intentions to engage in politics and to reduce one's carbon footprint. British Journal of Psychology, 105, 35-56. 
Jolley, D., \& Douglas, K. M. (2014b). The effects of anti-vaccine conspiracy theories on vaccination intentions. PLoS ONE, 9, e89177.

Jolley, D., \& Douglas, K. M. (2017). Prevention is better than cure: Addressing anti-vaccine conspiracy theories. Journal of Applied Social Psychology, 47(8), 459-469.

Jolley, D., Douglas, K., Leite, A., \& Schrader, T. (2019). Belief in conspiracy theories and intentions to engage in everyday crime. British Journal of Social Psychology. Advanced Online Publication.

Jonas, E., McGregor, I., Klackl, J., Agroskin, D., Fritsche, I., Holbrook, C., ... \& Quirin, M. (2014). Threat and defense: From anxiety to approach. In Advances in experimental social psychology (Vol. 49, pp. 219-286). Academic Press.

Jost, J. T., Hawkins, C. B., Nosek, B. A., Hennes, E. P., Stern, C., Gosling, S. D., \& Graham, J. (2014). Belief in a just God (and a just society): A system justification perspective on religious ideology. Journal of Theoretical and Philosophical Psychology, 34(1), 56.

Kahan, D. M., Peters, E., Dawson, E. C., \& Slovic, P. (2017). Motivated numeracy and enlightened self-government. Behavioural Public Policy, 1(1), 54-86.

Kane, M. J., Core, T. J., \& Hunt, R. R. (2010). Bias versus bias: Harnessing hindsight to reveal paranormal belief change beyond demand characteristics. Psychonomic Bulletin \& Review, 17(2), 206-212.

Klein, R. A., Ratliff, K. A., Vianello, M., Adams, R. B., Jr., Bahník, Š., Bernstein, M. J., ... Nosek, B. A. (2019, July 25). Investigating Variation in Replicability: A "Many Labs" Replication Project. Retrieved from osf.io/wx7ck

Kochenderfer-Ladd, B., \& Pelletier, M. E. (2008). Teachers' views and beliefs about bullying: Influences on classroom management strategies and students' coping with peer victimization. Journal of school psychology, 46(4), 431-453.

Lai, C. K., Marini, M., Lehr, S. A., Cerruti, C., Shin, J. E. L., Joy-Gaba, J. A., ... \& Frazier, R. S. (2014). Reducing implicit racial preferences: I. A comparative investigation of 17 interventions. Journal of Experimental Psychology: General, 143(4), 1765.

Lantian, A., Muller, D., Nurra, C., \& Douglas, K. M. (2016). Measuring belief in conspiracy theories: Validation of a French and English single-item scale. International Review of Social Psychology, 29, 1-14.

Lilienfeld, S. O., Ammirati, R., \& David, M. (2012). Distinguishing science from pseudoscience in school psychology: Science and scientific thinking as safeguards against human error. Journal of School Psychology, 50(1), 7-36.

Marchlewska, M., Cichocka, A., \& Kossowska, M. (2018). Addicted to answers: Need for cognitive closure and the endorsement of conspiracy beliefs. European Journal of Social Psychology, 48(2), 109-117.

McLaughlin, A. C., \& McGill, A. E. (2017). Explicitly teaching critical thinking skills in a history course. Science \& Education, 26(1-2), 93-105.

McLean, C. P., \& Miller, N. A. (2010). Changes in critical thinking skills following a course 
on science and pseudoscience: A quasi-experimental study. Teaching of Psychology, 37(2), 85-90.

Newheiser, A.-K., Farias, M., \& Tausch, N. (2011). The functional nature of conspiracy beliefs: Examining the underpinnings of belief in the Da Vinci Code conspiracy. Personality and Individual Differences, 51, 1007-1011.

Orne, M. T. (1962). On the social psychology of the psychological experiment: With particular reference to demand characteristics and their implications. American psychologist, 17(11), 776.

McGuire, W. J., \& Papageorgis, D. (1961). The relative efficacy of various types of prior belief-defense in producing immunity against persuasion. The Journal of Abnormal and Social Psychology, 62(2), 327.

Nisbett, R. E., Fong, G. T., Lehman, D. R., \& Cheng, P. W. (1987). Teaching reasoning. Science, 238(4827), 625-631.

Pashler, H., McDaniel, M., Rohrer, D., \& Bjork, R. (2008). Learning styles: Concepts and evidence. Psychological science in the public interest, 9(3), 105-119.

Pennycook, G., Cheyne, J. A., Seli, P., Koehler, D. J., \& Fugelsang, J. A. (2012). Analytic cognitive style predicts religious and paranormal belief. Cognition, 123(3), 335-346.

Pfau, M., Tusing, K. J., Koerner, A. F., Lee, W., Godbold, L. C., Penaloza, L. J., ... \& Hong, Y. H. (1997). Enriching the inoculation construct: The role of critical components in the process of resistance. Human Communication Research, 24(2), 187-215.

Primi, C., Morsanyi, K., Chiesi, F., Donati, M. A., \& Hamilton, J. (2016). The Development and Testing of a New Version of the Cognitive Reflection Test Applying Item Response Theory (IRT): A New Version of the Cognitive Reflection Test Applying IRT. Journal of Behavioral Decision Making, 29(5), 453-469

Richard, F. D., Bond Jr, C. F., \& Stokes-Zoota, J. J. (2003). One hundred years of social psychology quantitatively described. Review of General Psychology, 7(4), 331-363.

Roozenbeek, J., \& van der Linden, S. (2019). The fake news game: actively inoculating against the risk of misinformation. Journal of Risk Research, 22(5), 570-580.

Schielzeth, H., \& Nakagawa, S. (2013). Nested by design: model fitting and interpretation in a mixed model era. Methods in Ecology and Evolution, 4(1), 14-24.

Schustek, P., Hyafil, A., \& Moreno-Bote, R. (2019). Human confidence judgments reflect reliability-based hierarchical integration of contextual information. Nature communications, 10(1), 1-15.

Schwartz, L. M., Woloshin, S., Black, W. C., \& Welch, H. G. (1997). The role of numeracy in understanding the benefit of screening mammography. Annals of internal medicine, 127(11), 966-972.

Sidanius, J., \& Pratto, F. (2001). Social dominance: An intergroup theory of social hierarchy and oppression. Cambridge, UK: Cambridge University Press.

Simmons, J. P., Nelson, L. D., \& Simonsohn, U. (2011). False-positive psychology: 
Undisclosed flexibility in data collection and analysis allows presenting anything as significant. Psychological science, 22(11), 1359-1366.

Sorjonen, K., Falkstedt, D., Melin, B., \& Ingre, M. (2019). The peril of adjusting for baseline when using change as a predictor. Preprint. Available at https://psyarxiv.com/6p5hj/

Ståhl, T., \& van Prooijen, J. W. (2018). Epistemic rationality: Skepticism toward unfounded beliefs requires sufficient cognitive ability and motivation to be rational. Personality and Individual Differences, 122, 155-163.

Steegen, S., Tuerlinckx, F., Gelman, A., \& Vanpaemel, W. (2016). Increasing transparency through a multiverse analysis. Perspectives on Psychological Science, 11(5), 702-712.

Sutton, R. M., \& Douglas, K. M. (2014). Examining the monological nature of conspiracy theories, in: Power, politics, and paranoia: Why people are suspicious of their leaders (van Prooijen, J.W., \& van Lange, P.A., eds). Cambridge, UK: Cambridge University Press.

Svedholm, A. M., \& Lindeman, M. (2013). The separate roles of the reflective mind and involuntary inhibitory control in gatekeeping paranormal beliefs and the underlying intuitive confusions. British Journal of Psychology, 104(3), 303-319.

Swami, V., Coles, R., Stieger, S., Pietschnig, J., Furnham, A., Rehim, S., \& Voracek, M.

(2011). Conspiracist ideation in Britain and Austria: Evidence of a monological belief system and associations between individual psychological differences and real-world and fictitious conspiracy theories. British Journal of Psychology, 102(3), 443-463.

Swami, V., Voracek, M., Stieger, S., Tran, U. S., \& Furnham, A. (2014). Analytic thinking reduces belief in conspiracy theories. Cognition, 133, 572-585.

Twisk, J., Bosman, L., Hoekstra, T., Rijnhart, J., Welten, M., \& Heymans, M. (2018). Different ways to estimate treatment effects in randomised controlled trials. Contemporary clinical trials communications, 10, 80-85.

Van den Bulck, J., \& Custers, K. (2009). Belief in complementary and alternative medicine is related to age and paranormal beliefs in adults. European Journal of Public Health, 20(2), 227-230.

van Prooijen, J. W. (2017). Why education predicts decreased belief in conspiracy theories. Applied Cognitive Psychology, 31(1), 50-58.

Van Prooijen, J. W., Douglas, K. M., \& De Inocencio, C. (2017). Connecting the dots: Illusory pattern perception predicts belief in conspiracies and the supernatural. European Journal of Social Psychology.

Wesp, R., \& Montgomery, K. (1998). Developing critical thinking through the study of paranormal phenomena. Teaching of Psychology, 25(4), 275-278.

Whitson, J. A., \& Galinsky, A. D. (2008). Lacking control increases illusory pattern perception. Science, 322(5898), 115-117.

Whitson, J. A., Galinsky, A. D., \& Kay, A. (2015). The emotional roots of conspiratorial perceptions, system justification, and belief in the paranormal. Journal of Experimental Social Psychology, 56, 89-95.

World Medical Organization. (1964). Recommendations guiding physicians in biomedical research involving human subjects. Helsinki, Finland. 